\title{
IL-10 induces aberrant deletion of dendritic cells by natural killer cells in the context of HIV infection
}

\author{
Galit Alter, ${ }^{1}$ Daniel Kavanagh, ${ }^{1}$ Suzannah Rihn, ${ }^{1}$ Rutger Luteijn, ${ }^{1}$ David Brooks, ${ }^{2}$ \\ Michael Oldstone, ${ }^{3}$ Jan van Lunzen, ${ }^{4}$ and Marcus Altfeld ${ }^{1}$
}

${ }^{1}$ Ragon Institute of MGH, MIT, and Harvard, Charlestown, Massachusetts, USA. ${ }^{2}$ Department of Microbiology, Immunology, and Molecular Genetics and the UCLA AIDS Institute, David Geffen School of Medicine, UCLA, Los Angeles, California, USA. ${ }^{3}$ The Scripps Research Institute, San Diego, California, USA. ${ }^{4}$ Sektion Infektiologie, Med/Klinik I \& Ambulanzzentrum des UKE, Universitatsklinikum Hamburg-Eppendorf, Hamburg, Germany.

\begin{abstract}
Persistent levels of IL-10 play a central role in progressive immune dysfunction associated with chronic viral infections such as HIV, but the underlying mechanisms are poorly understood. Because IL-10 affects the phenotypic and functional properties of DCs, which are responsible for initiating adaptive immune responses, we investigated whether IL-10 induces changes in DC phenotype and function in the context of HIV infection. Here, we show that IL-10 treatment of immature and mature human DCs in culture induced contrasting phenotypic changes in these populations: immature DCs exhibited aberrant resistance to NK cell-mediated elimination, whereas mature DCs exhibited increased susceptibility to NKG2D-dependent NK elimination. Treatment of immature and mature DCs with HIV resulted in potent IL-10 secretion and the same phenotypic and functional changes observed in the IL-10-treated cells. Consistent with these in vitro data, LNs isolated from individuals infected with HIV exhibited aberrant accumulation of a partially "immature" DC population. Together, these data suggest that the progressive immune dysfunction observed in chronic viral infections might be caused in part by IL-10-induced reversal of DC susceptibility to NK cell-mediated elimination, resulting in the accumulation of poorly immunogenic DCs in LNs, the sites of adaptive immune response induction.
\end{abstract}

\section{Introduction}

DCs serve as immunological sentinels that monitor tissues for pathogens or tumor cells $(1,2)$. In tissues, DCs exist as immature, highly phagocytic cells that continuously sample the extracellular milieu for non-self antigens $(3,4)$. In this state, immature DCs express low levels of MHC class I (MHC-I) and MHC-II antigens. However, after tissue damage or encounter with a danger signal from a non-self antigen, a cascade of events occurs that allows DCs to shut down antigen uptake, upregulate antigen presentation, express MHC-I and -II molecules, migrate out of tissues, and begin to express costimulatory molecules required for the induction of adaptive immune responses (3). These events ensure that DCs efficiently prime an immune response that is responsible for clearance of the infection or tumor. In this capacity, DCs serve as the principal bridge between innate and adaptive immune responses (5).

Once mature, DCs express high levels of MHC in conjunction with an array of costimulatory molecules specifically tuned to potently induce $\mathrm{T}$ and $\mathrm{B}$ cell responses $(6,7)$. In contrast, immature DCs express low levels of MHC antigens and costimulatory molecules and have been shown to be poorly immunogenic, instead delivering strong tolerogenic signals $(8,9)$. Immature or incompletely mature DCs that aberrantly gain the potential to migrate to lymphoid organs have been implicated in a number of pathologic conditions, including asthma, cancer, and viral infections. Thus, as a result of the potent immunogenic or tolerogenic capacity of DCs, their function must be tightly regulated, and escape from this regulation can result in immunopathology $(4,9,10)$.

Conflict of interest: The authors have declared that no conflict of interest exists. Citation for this article: J Clin Invest. 2010;120(6):1905-1913. doi:10.1172/JCI40913.
NK cells are innate lymphocytes that patrol the body, surveying for tumor or infected cells that do not express self MHC-I $(11,12)$. In this capacity, NK cells are involved in the rapid elimination of autologous immature DCs that aberrantly enter the peripheral circulation, while sparing mature DCs, because of differences in MHC-I expression $(13,14)$. This NK cell-mediated quality control of DC populations ensures that only immunogenic mature DCs are able to gain access to inductive sites. Several groups have postulated that NK cells are involved in the tight control of DCs, as they regulate the DC populations that gain access to secondary lymphoid tissues by rapidly eliminating tolerogenic DCs that express low levels of MHC on their surface to prevent induction of tolerant responses (14-16).

NK cells express a wealth of receptors in different combinations $(17,18)$. These receptors can be either inhibitory or activating in nature, and it is therefore the balance of these opposing signals that dictates the activation state of an individual NK cell clone (19). Loss of MHC-I results in diminished inhibitory signals (which normally dominate), followed by an activating signal upon ligation of an activating receptor on the NK cell, required for the release of cytolytic granules or immunomodulators. Ferlazzo et al. have shown that activated NK cells eliminate immature DCs via the activating NK cell receptor NKp30 (16).

Several features of an effective immune response are altered in the context of chronic viral infections $(20,21)$. These include high levels of immune activation and generation of dysfunctional $\mathrm{T}$ cell responses (22-24). In the context of lymphocytic choriomeningitis virus (LCMV) infection, chronic elevated levels of the immunosuppressive cytokine IL-10 appear to be central to the observed inability of mice to control variants of the virus that establish chronic infection (LCMV clone 13; ref. 25). Blockade of 

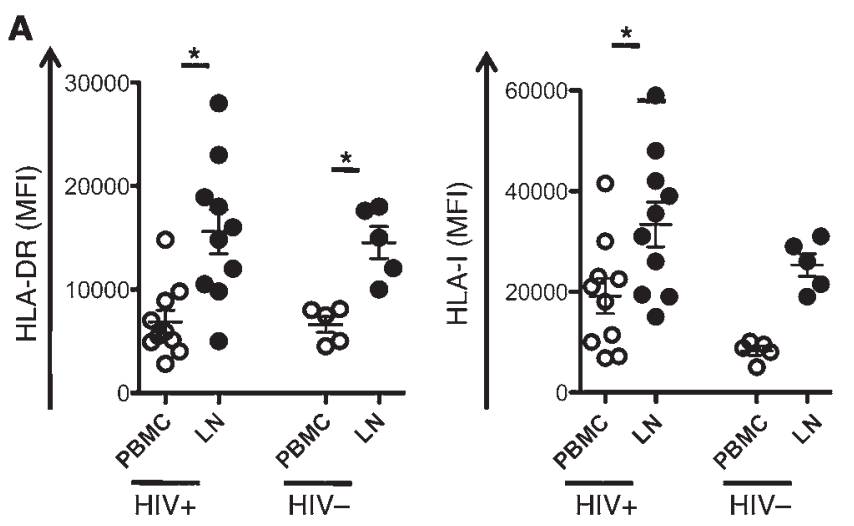

$\mathbf{B}$
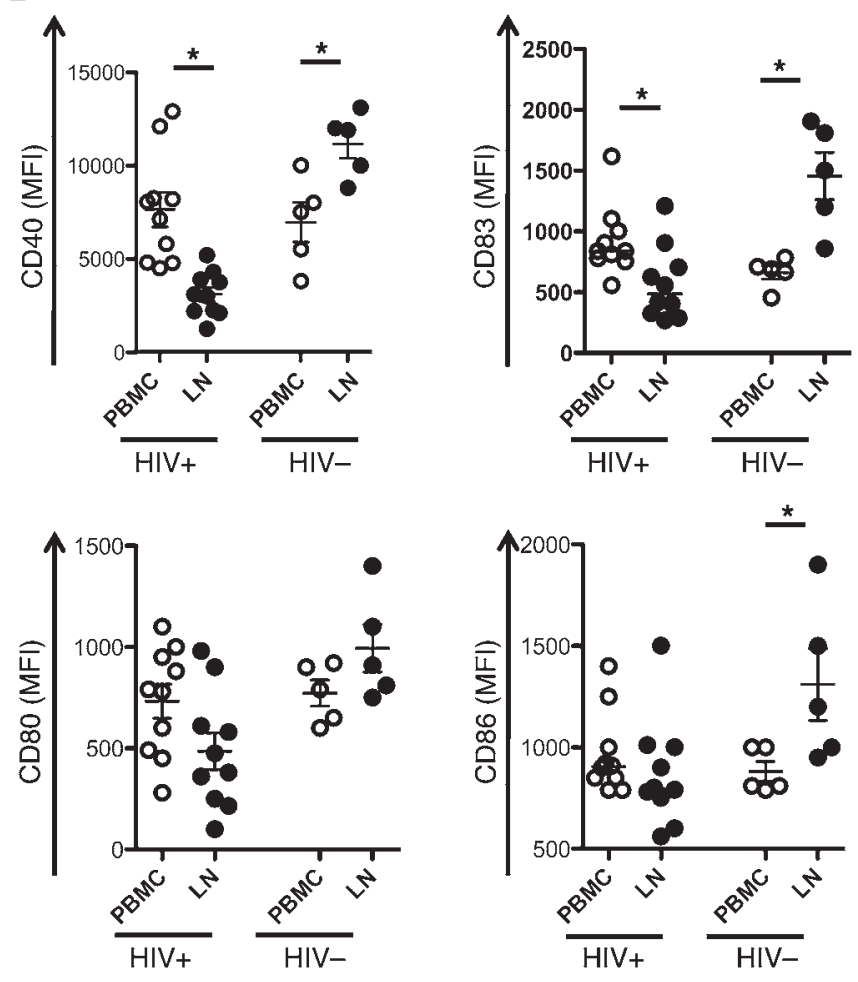

IL-10 during LCMV clone 13 infection resulted in resolution of the infection, demonstrating the critical role of this cytokine in skewing the antiviral immune response (26). Similarly, HIV infection is associated with elevated levels of IL-10 in the peripheral circulation, which suggests that this immunosuppressive cytokine may also contribute to lack of disease control in this viral infection. However, the mechanisms by which IL-10 skews the immune response are poorly understood.

IL-10 has been reported to have a profound impact on both phenotypic and functional properties of DCs, resulting in the differentiation of tolerogenic DCs that causes poor $\mathrm{T}$ cell induction (27-30). Interestingly, chronic LCMV infection has previously been associated with changes in DC phenotype in LNs that are reminiscent of tolerogenic DC accumulation (25); such changes are potentially responsible for the induction of dysfunctional adaptive responses. Given the critical nature of NK cell regulation of DC populations, we hypothesized that similar changes occur in the LN
Figure 1

Accumulation of aberrant DC populations in the LNs of HIV-infected individuals. Shown are mean \pm SEM of (A) HLA-DR and HLA-I (i.e., HLA-A, -B, or -C) and (B) costimulatory molecules CD40, CD83, CD80, and CD86 on the surface of CD3-CD14-CD56-CD11 ${ }^{-}$DCs in PBMCs and LNs from HIV-infected and HIV-negative donors. ${ }^{*} P<0.05$.

DCs of HIV-infected individuals and that these changes are attributable to IL-10-induced alterations in NK cell-mediated quality control of DCs. In the present study, we observed accumulation of phenotypically altered DCs in the LNs of HIV-infected subjects that exhibited a more immature-like tolerogenic phenotype. In addition, we demonstrated that HIV-induced IL-10 secretion resulted in profound changes in the phenotype of both immature and mature DC populations, rendering partially mature DCs resistant to deletion by NK cells while making mature DCs susceptible to NKG2D-mediated NK cell elimination. These effects of IL-10 likely disrupt the access of normal DC populations to inductive sites, which may have a profound impact on the quality of the antiviral adaptive immune response.

\section{Results}

Accumulation of partially mature DC populations in LNs from HIV-infected individuals. Chronic viral infections are associated with declining adaptive immune function $(20,21,31)$, including the accumulation of dysfunctional $\mathrm{T}$ cells that express inhibitory receptors on their surface, including programmed death receptor 1 (PD-1; ref. 31). However, it is uncertain whether the observed dysfunction is caused by direct effects of chronic viral replication on $\mathrm{T}$ cells or indirect mechanisms that act to induce poorly functional $\mathrm{T}$ cell responses. It is possible that early changes in the repertoire of DCs may result in the generation of progressively dysfunctional $\mathrm{T}$ cell responses, specifically at inductive sites. To test whether chronic HIV infection was associated with changes in DC populations in the peripheral circulation and LNs, we characterized the maturation phenotype of DCs in the peripheral blood (PB) and LNs of 10 individuals within the first year of untreated HIV infection as well as 5 HIV-negative controls. DCs were remarkably different in the PB compared with those in the LNs (Figure 1). Interestingly, DCs in the LNs exhibited significantly higher levels of HLA-DR and HLA class I (HLA-I) expression - indicating that these cells had received maturation signals - compared with those in $\mathrm{PB}$ $(P=0.001$ and $P=0.007$, respectively; Figure $1 \mathrm{~A})$. However, despite elevated levels of HLA molecules, these cells expressed significantly fewer of the costimulatory molecules CD40 and CD83 in the LNs of HIV-infected versus HIV-negative individuals $(P=0.001$ and $P=0.01$, respectively; Figure $1 \mathrm{~B}$ ). These data suggest that untreated early HIV infection is associated with aberrant accumulation of DCs that pass NK cell quality control because of their normal levels of MHC-I and -II, but exhibit a more immature/tolerogenic phenotype as a result of reduced levels of costimulatory molecules. These data potentially reflect earlier selection of aberrant DCs in vivo as a result of differential deletion of particular DC populations in the LN, or because of novel recruitment of these aberrant DCs after exposure to viral replication.

IL-10 modulates HLA-I expression on DCs. Central to the immunologic dysfunction observed in chronic viral infections, such as HIV, HCV, and LCMV, are persistent elevated levels of the immunoregulatory cytokine IL-10 (20,32). Previous work has demonstrated that in vitro 
A
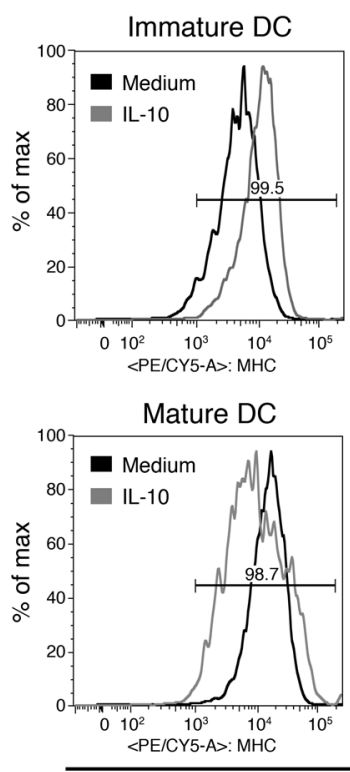

HLA-I
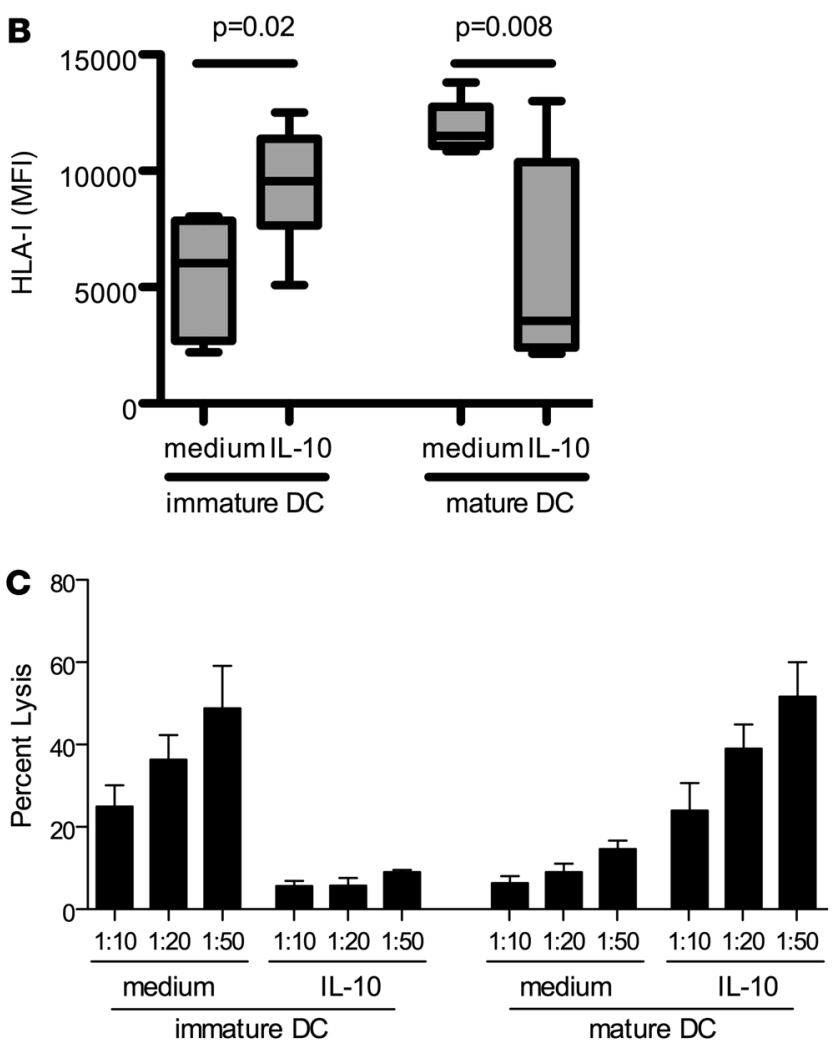

Figure 2

IL-10 reverses DC subset susceptibility to NK cell-mediated Iysis via altered MHC-I expression levels. (A) Changes in MHC-I (i.e., MHC-A, $-B$, or $-C$ ) expression on the surface of immature and mature monocyte-derived DCs in the presence of medium alone or IL-10. (B) Box and whisker plot denotes median and range of MHC-I expression on immature and mature DCs in the presence or absence of IL-10 $(n=6$ per group). (C) Mean \pm SEM percent lysis of immature or mature DCs at $1: 10,1: 20$, and 1:50 effector/target ratios with NK cells after coculture with medium alone or with IL-10 (using 6 independent donors). culture of both immature and mature DCs with IL-10 results in profound phenotypic changes (30). To characterize the effect of IL-10 on DC expression of MHC-I, we cocultured immature and mature monocyte-derived DCs in the presence or absence of IL-10 for 2 days and then measured MHC-I surface expression. As expected, MHC-I expression was significantly lower on immature than mature DCs in the absence of IL-10 ( $P=0.0002$; Figure 2 , A and $\mathrm{B})$. In the presence of IL-10, mature DCs lost MHC-I expression $(P=0.008)$; however, MHC-I level increased on the surface of IL-10-treated immature DCs compared with untreated immature DCs $(P=0.02$; Figure 2, A and B). Thus, IL-10 has opposite effects on MHC-I expression levels on immature and mature DCs, potentially rendering them differentially susceptible to NK cell-mediated lysis.

IL-10 modifies DC susceptibility to NK cell-mediated deletion. Given the profound differences in MHC-I expression on immature and mature DCs after treatment with IL-10, we tested the hypothesis that these changes render IL-10-treated DCs differentially susceptible to NK cell-mediated lysis. Thus, IL-10-treated and untreated immature and mature chromium-labeled DCs were cocultured with autologous NK cells for 6 hours. As reported previously, immature DCs were susceptible to NK cell-mediated lysis, whereas mature DCs were resistant to lysis (Figure 2C). After IL-10 treatment, immature DCs became resistant to NK cell-mediated elimination at all effector/target ratios compared with the untreated immature DCs $(P<0.05$, all comparisons; Figure $2 \mathrm{C})$. In contrast, IL-10 treatment rendered mature DCs susceptible to elimination by autologous NK cells compared with untreated mature DCs $(P<0.05$, all comparisons; Figure $2 \mathrm{C})$. Thus, it is likely that IL-10-induced changes in MHC-I expression renders immature DCs resistant while rendering mature DCs susceptible to elimination by autologous NK cells.
IL-10 skews DCs toward a tolerogenic phenotype. In addition to changes in MHC-I expression, we determined whether IL-10 treatment of immature and mature DCs affects the expression of other maturation markers. Similar to its effects on MHC-I, IL-10 slightly increased MHC-II expression on immature DCs, while downregulating it on mature DCs (Figure 3, A and B), as previously reported (30). Although MHC-II expression on immature DCs did not reach the levels expected based on ex vivo DCs observed in the LNs of HIVinfected individuals (Figure 1), in vitro treatment of DCs resulted in the upregulation of this molecule on these cells, which typically express little MHC-I. Thus, it is possible that in vivo immature DCs may be generated with a greater dynamic range of MHC-II expression, allowing NK cells to specifically select for those with higher levels of this molecule on their surface, or that additional signals in the LN may stimulate enhanced expression of MHC-II, but not of other molecules. Moreover, increasing concentrations of IL-10 appeared to increase the expression of MHC-II on the surface of immature DCs (Supplemental Figure 1; available online with this article; doi:10.1172/JCI40913DS1). This indicates that at higher concentrations of IL-10 - which may occur in particular microenvironments - immature DCs may upregulate this molecule to a greater extent. Furthermore, IL-10 treatment of immature DCs resulted in reduced expression of CD86, which suggests that despite the increase in HLA-DR, these DCs do not upregulate the required costimulatory molecules expressed on fully mature immunogenic DCs. Conversely, IL-10 stimulation of mature DCs resulted in reduced CD11c, CD83, CD86, CD40, and HLA-DR expression. Furthermore, IL-10 was able to alter both immature and mature DC phenotypes at even 100-fold lower concentrations of the immunoregulatory cytokine (Supplemental Figure 1), demonstrating the substantial impact of IL-10, even at low doses, on the phenotype of DCs. 
A
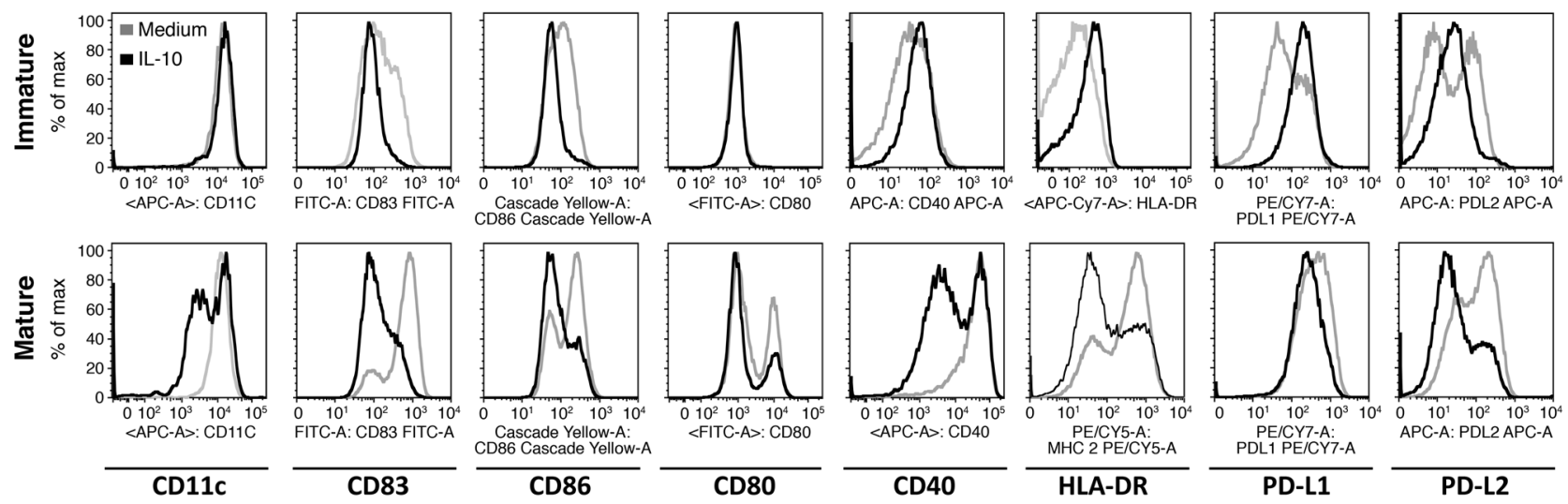

PDL1 PE/CY7-A

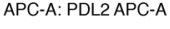

B
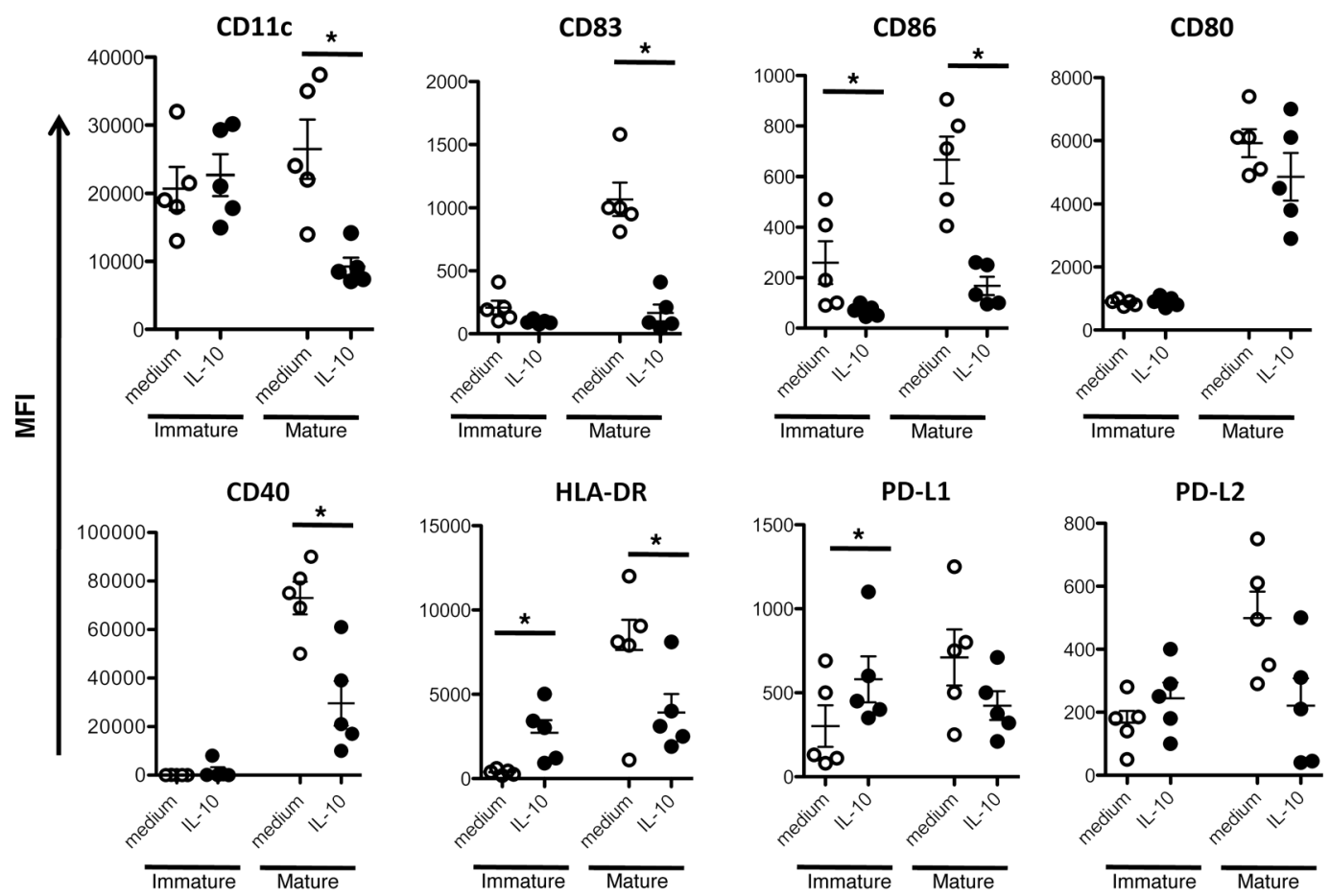

Figure 3

IL-10 treatment of immature and mature DCs results in a more tolerogenic DC phenotype. (A) Expression pattern of a range of phenotypic markers on the surface of immature and mature monocyte-derived DCs in the presence of medium alone or IL-10. (B) Mean \pm SEM of the phenotypic markers for all 5 experiments. ${ }^{*} P<0.05$.

Recently, 2 negative regulators of $\mathrm{T}$ cell function, programmed death ligand 1 (PD-L1) and PD-L2, have been implicated in regulating the quality of T cell responses induced by DCs. We therefore monitored IL-10-treated immature and mature DCs for changes in these 2 molecules. Immature DCs significantly upregulated PD-L1 $(P=0.004)$ and exhibited an overall shift in the expression of PD-L2 after treatment with IL-10 (Figure 3, A and B). Interestingly, PD-L1 and PD-L2 were both downregulated on mature DCs after treatment with IL-10. Thus, IL-10 treatment results in a partially mature tolerogenic phenotype on immature DCs.

NKG2D-dependent elimination of IL-10-treated DCs. NK cellmediated elimination of target cells is controlled by a delicate balance of inhibitory and activating signals (19). The loss of inhibitory signals tips the balance away from inhibition, but is not sufficient to induce killing by an NK cell; a second activating signal is critically required to induce the functional killing of the target cell. The activating C-type lectin NKG2D has been implicated in NK cell-mediated control of several viral infections and tumors (33). Given the multifunctional role of this activating NK cell receptor, we examined whether it could also be involved in activation of NK cells by IL-10-treated DCs. We stained IL-10-treated or untreated immature and mature DC populations with an NKG2D-fusion construct. The NKG2Dfusion construct did not bind to either of the immature DC populations (Figure 4A), which suggests that IL-10 was not inducing NKG2D ligands on the surface of immature DCs. 
A
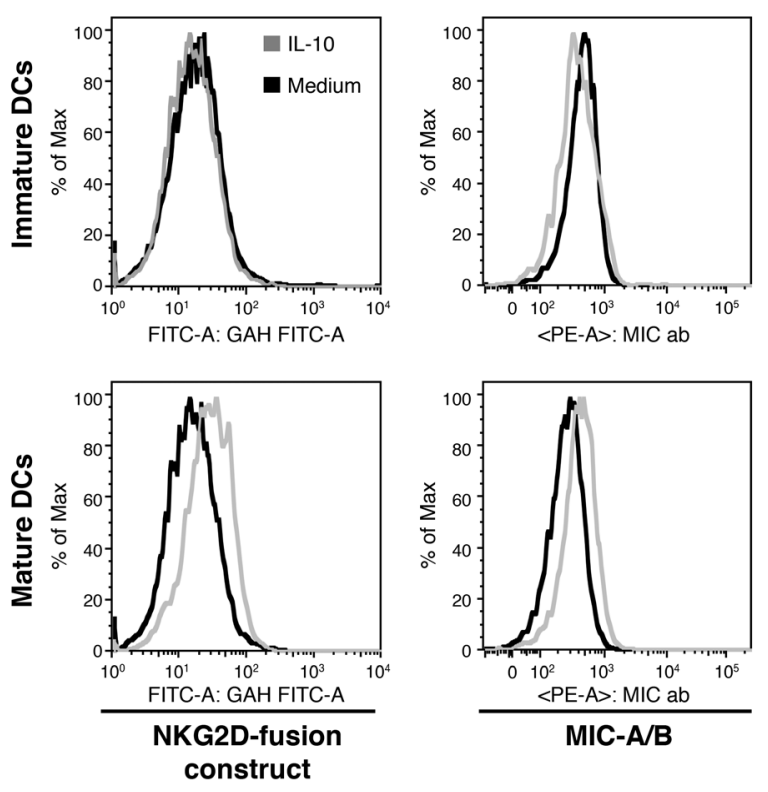

B

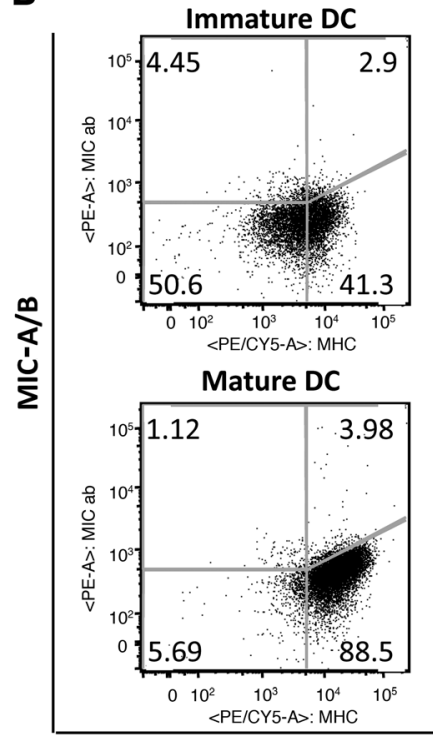

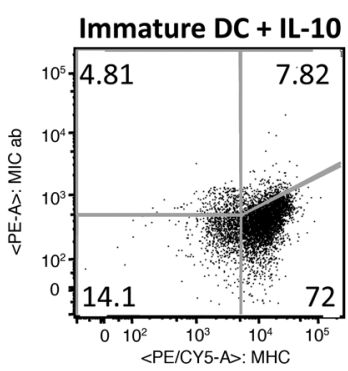

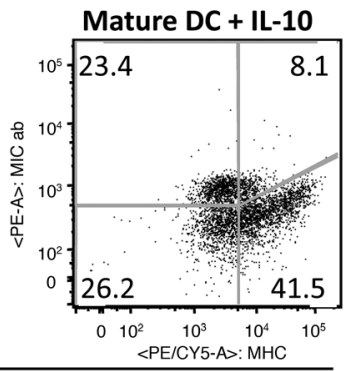

MHC-I
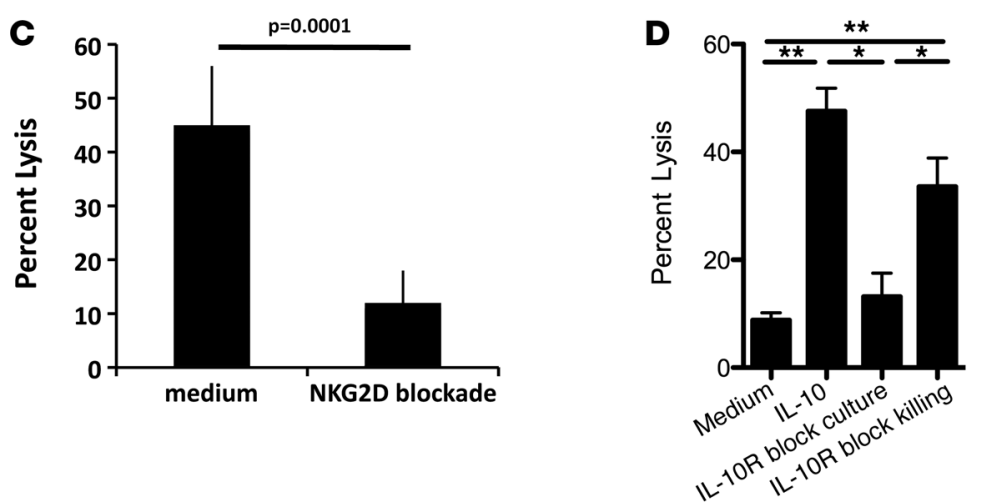

Figure 4

NK cells eliminate IL-10-treated mature DCs in an NKG2D-dependent manner, which is blocked by IL-10R blockade. (A) Binding capacity of an NKG2D-fusion construct and expression of MIC-A/B on immature and mature DCs in the presence of medium alone or IL-10. (B) Coexpression levels of MIC-A/B and MHC-I on the surface of immature or mature DCs in the presence and absence of IL-10. (C) Mean \pm SEM capacity of NK cells to lyse IL-10-treated mature DCs in the absence or presence of an NKG2D-blocking antibody $(n=5)$. (D) Mean \pm SEM percent lysis of mature DCs by autologous NK cells after maturation in the presence of medium alone, IL-10 alone, and IL-10 plus IL-10R-blocking antibody — either during maturation in vitro (culture) or during the NK cell/DC killing assay (killing). ${ }^{\star} P<0.05 ;{ }^{* \star} P<0.01$.

However, the NKG2D-fusion construct bound to the IL-10treated mature DC population, but not the untreated mature DCs, which demonstrated that NKG2D ligands are upregulated on the surface of mature DCs only after IL-10 treatment.

NKG2D binds to a number of different stress-inducible ligands, including $\mathrm{MHC}-1$ homolog A (MIC-A), MIC-B, and UL-16-binding proteins (ULBP-1-ULBP-3; ref. 33). To identify whether MIC-A or -B were upregulated on the surface of IL-10-treated mature DCs, we stained IL-10-treated and untreated immature and mature DCs with antibodies against MIC-A/B. Although the antibody did not bind to either of the immature DC populations, it did bind to the IL-10-treated mature DCs, but not to IL-10-treated immature DCs (Figure 3B). These data suggest that the MIC-A and MIC-B are upregulated on mature, but not immature, DCs after treatment with IL-10.
As the upregulation of MIC-A/B was modest on the whole IL-10treated mature DC population, we sought to ascertain whether there was any relation between DCs that upregulate MIC-A/B expression and those that lose MHC-I expression, as these would represent the most vulnerable population of DCs for NK cell-mediated elimination. We costained the different DC populations for MIC-A/B and MHC-I simultaneously in the presence or absence of IL-10 in 4 separate individuals (Figure 4B). The level of MHC-I increased remarkably on the surface of IL-10-treated immature DCs, in the absence of any changes in MIC-A/B levels (Figure 4B). In contrast, MHC-I expression declined dramatically in nearly half the mature DCs in the presence of IL-10, of which nearly half potently upregulated MIC-A/B (Figure 4B). These data suggest that the NKG2D ligands MIC-A and MIC-B are upregulated specifically on IL-10-treated mature DCs that have lost MHC-I expression. 

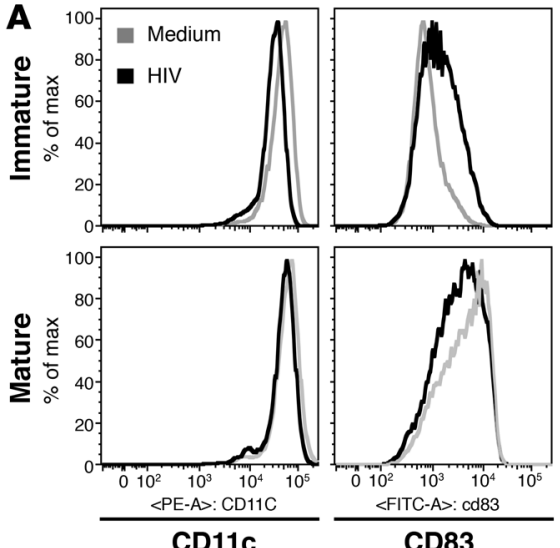

CD83
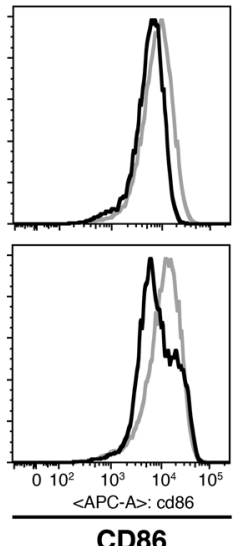
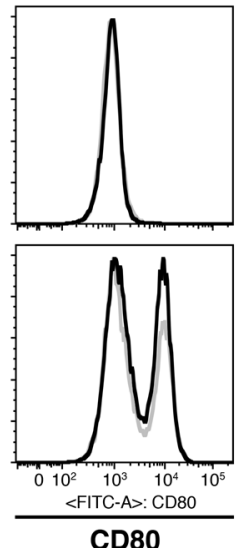
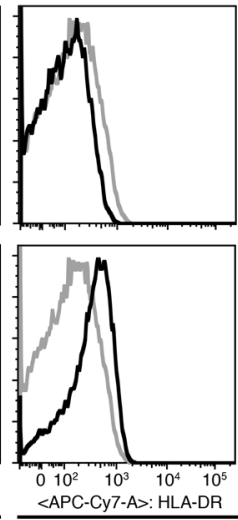

HLA-DR
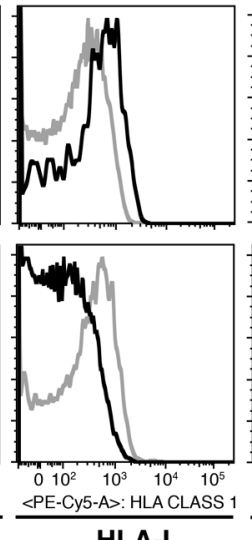

HLA-I
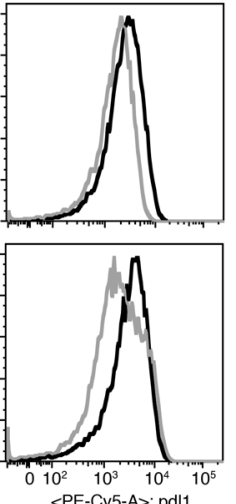

PD-L1
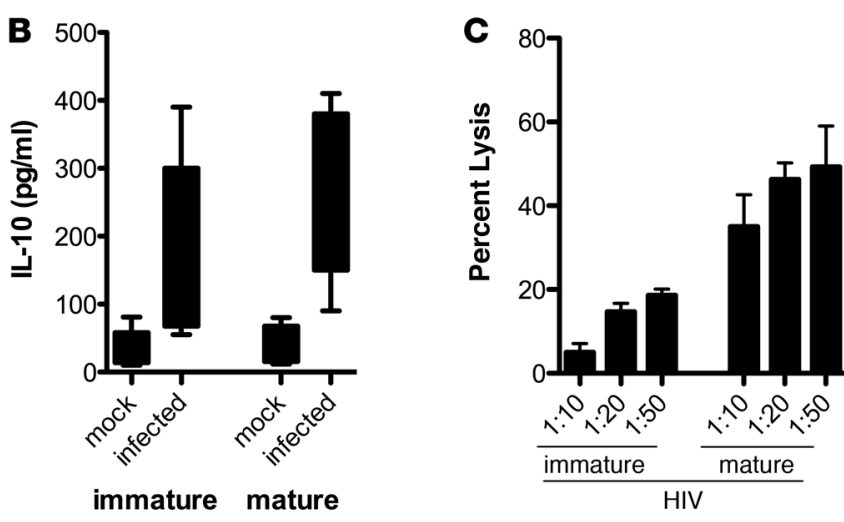

NK cell-induced lysis of IL-10-treated mature DCs is mediated by NKG2D. The above data demonstrated that IL-10 treatment of DCs results in the upregulation of NKG2D ligands and downregulation of MHC-I on the surface of mature DCs. To define the potential role of these NKG2D ligands in NK cell-mediated lysis of autologous IL-10-treated mature DCs, we attempted to measure the involvement of NKG2D in NK cell-mediated DC killing by blocking this receptor with a blocking antibody. Whereas IL-10-treated mature DCs were lysed efficiently, NKG2D blockade prior to coculture with autologous IL-10-treated mature DCs resulted in a nearly $80 \%$ reduction in NK cell-mediated killing of autologous DCs $(P=0.0001$; Figure $4 C)$. Thus, NKG2D-dependent activation of NK cells constitutes a principal mechanism by which NK cells recognize and eliminate IL-10 treated mature DCs.

$I L-10$ receptor blockade during coculture restores mature $D C$ resistance to NK cell-mediated elimination. To definitively determine whether IL-10 is responsible for modulating DC susceptibility to NK cell-mediated elimination, as well as to define at which step IL-10 blockade reverses mature DC susceptibility to NK cell-mediated elimination, mature DCs were treated with an IL-10 receptor-blocking (IL-10R-blocking) antibody, either during maturation in vitro or during the NK cell/DC killing assay. Although IL-10R blockade had no impact on mature DC elimination during the chromium release assay, treatment of mature DCs with the IL-10R-blocking antibody during the maturation process dramatically reduced DC susceptibility to elimination by autologous NK cells (Figure 4D). These data strongly suggest that IL-10 renders mature DCs susceptible to NK cell-mediated lysis indirectly via alterations in DC phenotype rather than through potentiation of NK cell-mediated lysis of DCs during coculture.

\section{Figure 5}

Coculture of DCs with HIV results in IL-10 secretion and an altered DC phenotype. (A) Expression patterns of phenotypic markers on the surface of immature and mature monocyte-derived DCs in the presence of medium alone or HIV JRCSF. (B) Range of IL-10 secretion measured by ELISA in the supernatant of immature and mature DCs cocultured in the presence of or absence of HIV JRCSF $(n=5)$. (C) Mean \pm SEM percent lysis of immature and mature DCs at 1:10, 1:20, and 1:50 effector/target ratios with NK cells after coculture with HIV (using 5 independent donors).
HIV alters DC maturation and phenotype analogously to IL-10. To assess whether the observed differences in IL-10-mediated DC phenotypic modifications are also observed after DC coculture with HIV, we characterized the surface expression of the above markers on both immature and mature monocyte-derived DCs cultured in the presence or absence of an R5 virus. Coculture with HIV induced partial maturation of immature DCs, resulting in elevated levels of CD83, HLA-DR, and HLA-I expression, but not CD80 or CD86 expression, on immature DCs (Figure $5 \mathrm{~A})$. Remarkably, this phenotype of HIV-treated immature DCs was similar to the observed DC populations in the LNs of HIVinfected individuals (Figure 1). In contrast, HIV-treated mature DCs exhibited reduced expression of CD83, CD86, HLA-DR, and HLA-I (Figure 5A). Additionally, both immature and mature DCs upregulated PD-L1 after coculture with HIV. Furthermore, coculture of DCs with HIV resulted in potent secretion of IL-10 by both subsets of DCs (Figure 5B), as previously described (34), and supernatant from these cocultures induced similar changes in DC phenotype (data not shown). These data suggest that HIV-induced modulation of DC phenotype may be attributable in part to autocrine IL-10-induced effects.

To determine whether monocyte-derived DC exposure to HIV renders immature and mature DCs differentially susceptible to NK cell-mediated elimination, we performed a similar NK cell/DC killing assay in the presence of HIV. Immature and mature DCs were cocultured for 2 days in the presence of HIV, then placed in coculture with autologous NK cells. As we showed for IL-10 treatment of DCs, HIV pretreatment resulted in an inverted susceptibility of DCs for elimination by NK cells: immature DCs were resis- 
tant to elimination, whereas mature DCs were robustly eliminated by autologous NK cells (Figure 5C). These data demonstrate that HIV itself, which induces IL-10 secretion from DCs, can reverse DC susceptibility to NK cell-mediated elimination.

\section{Discussion}

Chronic viral infections are characterized by the development of progressive T cell dysfunction $(21,31)$, and IL-10 has been recently implicated in this process (25). However, the mechanisms by which IL-10 induces this dysfunction are still poorly understood. Here we show that IL-10 reversed the susceptibility of immature and mature DCs for NK cell-mediated deletion. Whereas NK cells are normally involved in the elimination of immature DCs and the preservation of immunogenic mature DCs $(13,14)$, coculture with IL-10 or HIV resulted in the rapid elimination of mature DCs, while immature DCs were spared. This IL-10/HIV-mediated redistribution was also observed ex vivo in LNs from HIV-infected individuals, in such a way that HIV infection was associated with accumulation of DCs with normal MHC-I and -II expression, but also with reduced levels of costimulatory molecules, potentially reflective of an aberrant selection of a more immature/ tolerogenic population of DCs prior to entry into the LN. Interestingly, IL-10/HIV-induced elimination of mature DCs was attributable to downregulation of MHC-I and upregulation of MIC-A/B. In contrast, in the presence of IL-10/HIV, poorly immunogenic immature DCs upregulated MHC-I and were protected from NK cell-mediated elimination. Thus, IL-10-induced immune dysfunction may be related, at least in part, to the modification of DC populations able to gain access to inductive sites, where they may profoundly modulate the quality of the adaptive immune response.

The tolerogenic impact of IL-10 has been illustrated in several recent studies $(35,36)$. Most notably, the delivery of an antigen in the presence of IL-10 results in antigen-specific tolerance (30). In addition, ex vivo IL-10 treatment of freshly isolated APCs (myeloid DCs) has been shown to alter APC phenotype, downregulating proliferation and differentiation of Th1 cells in vivo (35) as well as in vitro (30). The immunosuppressive activity of IL-10 has been linked to blockade of proinflammatory cytokine production, downregulation of antigen presentation, downregulation of costimulation, and reduced expression of costimulatory molecules (35). In the present study, we evaluated IL-10-induced phenotypic changes on DCs and how these relate to alteration in DC susceptibility to NK cell-mediated elimination. IL-10 treatment resulted in a dramatic alteration in the phenotype of both immature and mature DCs. After coculture with IL-10, both mature and immature DCs expressed low levels of costimulatory molecules (Figure 3); however, only mature DCs lost MHC-I and -II expression (Figure 2). In contrast, the immature DCs upregulated MHC molecules potently after exposure to IL-10, which suggests that immature and mature DCs respond differentially to the same immunosuppressive cytokine.

Previous work has shown that NK cells eliminated immature DCs robustly via NKp30 (16), while sparing mature DCs $(13,14)$, but not via NKG2D. Here we showed that IL-10 reversed the susceptibility of immature and mature DCs for NK cell-mediated deletion. IL-10 treatment resulted in the robust protection of immature DCs from elimination by autologous activated NK cells. In contrast, mature DCs upregulated NKG2D ligands and were rapidly eliminated in an NKG2D-dependent manner by autologous activated NK cells (Figure 4). These changes in DC susceptibility to NK cell-mediated elimination were reflected by aberrant accumulation of immature-like DCs, with elevated expression of
MHC-I in ex vivo LNs from HIV-infected individuals compared with those from HIV-negative individuals (Figure 1). Furthermore, HIV induced IL-10 secretion by DCs and led to similar changes in HLA-I and NKG2D ligand expression on DCs (Figures 4 and 5), which suggests that HIV may induce these aberrant changes in DC phenotype via upregulation of IL-10, which acts in an autocrine manner on producing cells (Figure 5). Chronic HIV infection is associated with a gradual accumulation of anergic CD3-CD56- ${ }^{-} \mathrm{NK}$ cells that lose the capacity to kill $(37,38)$; however, the observed redistribution of DC subsets was detectable as early as the first year of HIV infection, before the observed accumulation of these functionally exhausted NK cells. This finding suggests that although NK cell-mediated deletion may wane over time, these early changes may occur rapidly, contributing to early changes in the functional quality of emerging adaptive immune responses. Thus, HIV induced IL-10 production may result in the early aberrant accumulation of immature poorly immunogenic DCs that may be responsible for priming inefficient $\mathrm{T}$ cell responses.

Several studies have shown that immature DCs, which aberrantly gain access to inductive sites, deliver tolerogenic signals as a result of low-level expression of costimulatory molecules $(9,10)$. Here we showed that IL-10 treatment resulted in the upregulation of HLA-DR and MHC-I on immature DCs, but not in the expression of CD40, CD80, CD83, and CD86, costimulatory molecules required for efficient priming of $\mathrm{T}$ cell responses (Figure 3). Similarly, DCs in the LNs of HIV-infected individuals also exhibited a more immature phenotype, with low-level expression of costimulatory molecules (Figure 1); however, these DCs exhibited higher levels of MHC-I and -II expression compared with immature treated DC populations (Figures 3 and 5). This discrepancy may reflect NK cell-mediated selection of tolerogenic DCs with normal expression of MHC-I and -II molecules (which may be induced in vivo and possibly at higher doses of IL-10) or could be due to additional signals other than IL-10 that may occur in vivo and cumulatively alter the phenotype of DCs during infection. Interestingly, IL-10 treatment of immature DCs also led to the upregulation of PD-L1 and PD-L2, expressed on the surface of exhausted T cells (Figure 3). PD-L1/L2 have also been shown to be induced via the stimulation of DCs with HIVencoded TLR7/8 ligands (39), which are also responsible for the upregulation of IL-10 (40). Thus, the combined lack of expression of costimulatory molecules, along with the upregulation of inhibitory ligands, may amplify the induction of tolerogenic $\mathrm{T}$ cell responses. These data are consistent with a model in which HIV-induced, IL-10mediated changes in DC populations in the LNs of HIV-infected individuals may contribute to induction of dysfunctional adaptive immune responses unable to clear or contain the viral infection.

Serum levels of IL-10 increase rapidly following several acute viral infections $(41,42)$. The synergistic induction of both proinflammatory and this antiinflammatory stimuli may play a profound role in the general regulation of the potency and duration of the antiviral response. However, the mechanism by which IL-10 mediates its effects early in infection is not understood. Based on the results presented here, it is plausible that early IL-10 may be involved in limiting the window of time in which DCs are able to gain access to inductive sites, by regulating DC susceptibility for NK cell-mediated elimination. Thus, as IL-10 accumulates, DCs leaving tissues may become more vulnerable to NK cell-mediated elimination with progressive infection, limiting the number of DCs that gain access to $\mathrm{LN}$. Therefore, IL-10 may recruit the activity of NK cells to regulate the size of the adaptive immune response during early infection. 
Based on our present findings, we propose a mechanism by which IL-10 induces immune dysregulation in persistent viral infections, using HIV infection as a model. Exposure of DCs to HIV resulted in secretion of large quantities of IL-10 that significantly modulated DC phenotype in an autocrine manner, resulting in the rapid elimination of mature DCs in an NKG2D-dependent manner. In contrast, immature DCs upregulated MHC-I and were protected from deletion by DCs, resulting in their accumulation in LNs, where they may induce dysfunctional adaptive immune responses. Further investigation is warranted to define qualitative differences in adaptive immune priming by these aberrant DC subsets that accumulate in the LN during early HIV infection. However, given this aberrant deletion of DCs in an NKG2D-dependent manner by NK cells, it is plausible that new targeted therapies aimed at blocking NKG2D-dependent elimination of mature DCs early in HIV infection may reduce the elimination of mature DCs that would still have the potential to induce higher-quality $\mathrm{T}$ cell responses required for antiviral control. This strategy may also overcome some of the more generalized effects of IL-10 blockade and may result in specific amelioration of the immune defects induced by prolonged exposure to virus replication.

\section{Methods}

Subjects. A total of 26 subjects (10 HIV infected and 16 HIV negative) was recruited for this study. The $10 \mathrm{HIV}$-infected subjects were recruited in early HIV infection (i.e., within the first year of infection), prior to initiation of antiretroviral therapy. For all HIV-infected individuals, at least 1 PBMC and at least $1 \mathrm{LN}$ time point was obtained during the first year of HIV infection. The average viral load for these individuals was $2.4 \times 10^{6} \mathrm{RNA}$ copies $/ \mathrm{ml}$ (range, $5 \times 10^{1}$ to $3.5 \times 10^{6}$ copies $/ \mathrm{ml}$ ), and the average $\mathrm{CD} 4^{+} \mathrm{T}$ cell count was 419 cells/ $\mu \mathrm{l}$ (range, 175 to 810 cells/ $\mu \mathrm{l}$ ). The local and Massachusetts General Hospital Institutional Review Boards approved the study, and each subject gave written informed consent.

LN biopsies. Axillary LN biopsies were performed under local anesthesia after written informed consent was obtained. $\mathrm{LN}$ samples were placed in normal saline at $4{ }^{\circ} \mathrm{C}$ and immediately transferred to the laboratory for further processing. Mononuclear cells were mechanically liberated from lymphoid tissue, and LN mononuclear cells were frozen in FCS plus 10\% DMSO. Fresh PBMCs were separated from PB by density gradient centrifugation using Ficoll-Hypaque (Sigma-Aldrich) and frozen in FCS plus 10\% DMSO.

Generation of monocyte-derived DCs. PBMCs and plasma were isolated using standard Ficoll separation from the blood of HIV-negative donors. Plasma was sterile filtered using steriflip (Millipore Corp.). PBMCs were washed and incubated in a $75-\mathrm{cm}^{2}$ cell culture flask at $37^{\circ} \mathrm{C}\left(5 \% \mathrm{CO}_{2}\right)$. After 1 hour, the cell layer was pressure washed with warm HBSS (Mediatech Inc.) to remove nonadhering cells. Cells were incubated for 5 days in RPMI, P/L, HEPES, and 1\% autologous human serum. At days 0, 2, and 4, IL-4 and GM-CSF (CellGenix) were added to final concentrations of 20 and $10 \mathrm{ng} / \mathrm{ml}$, respectively. At day 5 , cells were incubated at $4{ }^{\circ} \mathrm{C}$ for 30 minutes, supernatant was harvested, and the remaining cells were collected by washing 3 times with ice-cold PBS. Maturation was performed subsequently for 2 days in the presence of $5 \mathrm{ng} / \mathrm{ml}$ tumor necrosis factor- $\alpha, 5 \mathrm{ng} / \mathrm{ml} \mathrm{IL-} 1 \beta, 150 \mathrm{ng} / \mathrm{ml} \mathrm{IL-} 6$, and $1,000 \mathrm{ng} / \mathrm{ml}$ prostaglandin-E2 (R\&D Systems). Both immature and mature DCs were cultured thereafter in the presence or absence of IL-10 at $40 \mathrm{ng} / \mathrm{ml}$ (R\&D Systems) and in some instances a IL-10R-blocking antibody (Biolegend).

DC maturation phenotype. The maturation status of myeloid DCs was evaluated by flow cytometry after maturation in the presence or absence of $40 \mathrm{ng} / \mathrm{ml} \mathrm{IL-10} \mathrm{(R \& D} \mathrm{Systems)} \mathrm{or} 10^{5}$ infectious units of HIV. DCs were stained with 2 different panels of antibodies. Panel 1 included biotinconjugated CD80, FITC-conjugated CD83, allophycocyanin-conjugated CD40, PE-Cy5.5-conjugated CD11c, PE-Cy5-conjugated MHC-I, allophycocyanin-Cy7-conjugated HLA-DR, PE-conjugated MIC-A/B, and Pacific Blue-conjugated CD3-CD14-CD19 (BD Biosciences). Panel 2 included PE-Cy7-conjugated PD-L1, allophycocyanin-conjugated PD-L2, PE-Cy5conjugated MHC-I, allophycocyanin-Cy7-conjugated HLA-DR, Pacific Blue-conjugated CD3-CD14-CD19 (BD Biosciences), and PE-Cy5.5-conjugated CD11c (Invitrogen). Cells were then washed, and a streptavidincascade yellow was added to Panel 1. Panel 1 was washed once more, after which all tubes were resuspended in $200 \mu \mathrm{l}$ of $2 \%$ paraformaldehyde until they were acquired on a flow cytometer. At least $3 \times 10^{5}$ cells were acquired, and data were analyzed using Flowjo.

NK cell enrichment. Pure NK cell populations were generated from whole blood by negative selection using the Stem Cell Rosette Sep cocktail (Stem Cell Technologies). Whole blood was incubated for 20 minutes in the presence of the cocktail, and pure NK cells were collected following Ficoll separation. The average purity of bulk NK cell populations was $93.8 \%$ (range, $92.4 \%$ to $97.5 \%$ ). Bulk NK cells were washed twice and resuspended at $10 \times 10^{6} \mathrm{cells} / \mathrm{ml}$ in RPMI 1640 containing 10\% heat-inactivated FBS, $2 \mathrm{mM} \mathrm{L-glutamine,} \mathrm{and}$ $50 \mathrm{IU} / \mathrm{ml}$ penicillin supplemented with $50 \mathrm{U} / \mathrm{ml} \mathrm{IL}-2$.

Chromium release assay. The ability of NK cells to lyse autologous DCs was examined using a standard chromium release assay. Mature and immature DCs, pulsed with IL-10, HIV, or neither, were labeled with $50 \mu \mathrm{Ci}$ $\mathrm{Na}_{2}\left(51 \mathrm{CrO}_{4}\right)\left(\mathrm{New}\right.$ England Nuclear) for 1 hour at $37^{\circ} \mathrm{C}, 5 \% \mathrm{Co}_{2}$. The target cells were washed 3 times and then placed in coculture with autologous NK cells at effector/target ratios of 50:1, 25:1, and 10:1 in the presence or absence of an IL-10R-blocking antibody (Biolegend). Supernatant was harvested onto a filter membrane plate after a 6 -hour incubation at $37^{\circ} \mathrm{C}$ and $5 \% \mathrm{CO}_{2}$. The percent lysis was calculated as (sample count - spontaneous release)/(maximal release - spontaneous release).

Statistics. To test for differences in the mean among several populations, an ANOVA with a Tukey's correction was used for all comparisons with $P$ values less than 0.05 . Error bars represent SD. A $P$ value less then 0.05 was considered significant.

\section{Acknowledgments}

This work was supported by the NIH (grant PO1 AI074415) and the Bill and Melinda Gates Foundation.

Received for publication August 21, 2009, and accepted in revised form March 3, 2010.

Address correspondence to: Galit Alter, Ragon Institute of MGH, MIT, and Harvard, Massachusetts General Hospital, 149 13th Street, Boston, Massachusetts 02129, USA. Phone: 617.724.0546; Fax: 617.726.5411; E-mail: galter@partners.org.
1. Steinman RM, Nussenzweig MC. Dendritic cells: features and functions. Immunol Rev. 1980;53:127-147.

2. Steinman RM, Witmer-Pack M, Inaba K. Dendritic cells: antigen presentation, accessory function and clinical relevance. Adv Exp Med Biol. 1993;329:1-9.

3. Steinman RM, Pack M, Inaba K. Dendritic cell development and maturation. Adv Exp Med Biol. 1997;417:1-6.

4. Hawiger D, et al. Dendritic cells induce peripheral
T cell unresponsiveness under steady state conditions in vivo. J Exp Med. 2001;194(6):769-779.

5. Mellman I, Turley SJ, Steinman RM. Antigen processing for amateurs and professionals. Trends Cell Biol. 1998;8(6):231-237.

6. Inaba K, Metlay JP, Crowley MT, Witmer-Pack M, Steinman RM. Dendritic cells as antigen presenting cells in vivo. Int Rev Immunol. 1990;6(2-3):197-206.

7. Breart B, Bousso P. Cellular orchestration of T cell priming in lymph nodes. Curr Opin Immunol. 2006; 18(4):483-490.

8. Steinman RM, et al. Dendritic cell function in vivo during the steady state: a role in peripheral tolerance. Ann N Y Acad Sci. 2003;987:15-25.

9. Steinman RM, Hawiger D, Nussenzweig MC. Tolerogenic dendritic cells. Annu Rev Immunol. 2003;21:685-711.

10. Thomson AW, Robbins PD. Tolerogenic dendritic 
cells for autoimmune disease and transplantation. Ann Rheum Dis. 2008;67(suppl 3):iii90-iii96.

11. Moretta A, Bottino C, Mingari MC, Biassoni R, Moretta L. What is a natural killer cell? Nat Immunol. 2002;3(1):6-8.

12. Moretta L, Bottino C, Pende D, Mingari MC, Biassoni R, Moretta A. Human natural killer cells: their origin, receptors and function. Eur J Immunol. 2002;32(5):1205-1211.

13. Gerosa F, Baldani-Guerra B, Nisii C, Marchesini V, Carra G, Trinchieri G. Reciprocal activating interaction between natural killer cells and dendritic cells. J Exp Med. 2002;195(3):327-333.

14. Gerosa F, et al. The reciprocal interaction of NK cells with plasmacytoid or myeloid dendritic cells profoundly affects innate resistance functions. J Immunol. 2005;174(2):727-734.

15. Ferlazzo G, Munz C. Dendritic cell interactions with NK cells from different tissues. J Clin Immunol. 2009;29(3):265-273.

16. Ferlazzo G, Tsang ML, Moretta L, Melioli G, Steinman RM, Munz C. Human dendritic cells activate resting natural killer (NK) cells and are recognized via the NKp30 receptor by activated NK cells. J Exp Med. 2002;195(3):343-351.

17. Biassoni R, et al. Human natural killer cell receptors and co-receptors. Immunol Rev. 2001;181:203-214.

18. Lanier LL. NK cell receptors. Annu Rev Immunol. 1998;16:359-393.

19. Lanier LL. Up on the tightrope: natural killer cell activation and inhibition. Nat Immunol. 2008; 9(5):495-502.

20. Brooks DG, McGavern DB, Oldstone MB. Reprogramming of antiviral $\mathrm{T}$ cells prevents inactivation and restores $\mathrm{T}$ cell activity during persistent viral infection. J Clin Invest. 2006;116(6):1675-1685.

21. Wherry EJ, et al. Molecular signature of CD8+ $\mathrm{T}$ cell exhaustion during chronic viral infection.
Immunity. 2007;27(4):670-684.

22. Cadogan M, Dalgleish AG. Pathogenesis of HIV: non-specific immune hyperactivity and its implications for vaccines. Clin Med. 2008;8(3):267-271.

23. Appay V, Sauce D. Immune activation and inflammation in HIV-1 infection: causes and consequences. J Pathol. 2008;214(2):231-241.

24. El-Far M, et al. T-cell exhaustion in HIV infection. Curr HIV/AIDS Rep. 2008;5(1):13-19.

25. Brooks DG, Trifilo MJ, Edelmann KH, Teyton L, McGavern DB, Oldstone MB. Interleukin-10 determines viral clearance or persistence in vivo. Nat Med. 2006;12(11):1301-1309.

26. Brooks DG, Lee AM, Elsaesser H, McGavern DB, Oldstone MB. IL-10 blockade facilitates DNA vaccine-induced $\mathrm{T}$ cell responses and enhances clearance of persistent virus infection. J Exp Med. 2008;205(3):533-541.

27. Fiorentino DF, et al. IL-10 acts on the antigen-presenting cell to inhibit cytokine production by Th1 cells. J Immunol. 1991;146(10):3444-3451.

28. de Waal Malefyt R, et al. Interleukin 10 (IL-10) and viral IL-10 strongly reduce antigen-specific human $\mathrm{T}$ cell proliferation by diminishing the antigenpresenting capacity of monocytes via downregulation of class II major histocompatibility complex expression. J Exp Med. 1991;174(4):915-924.

29. Willems F, et al. Interleukin-10 inhibits B7 and intercellular adhesion molecule-1 expression on human monocytes. Eur J Immunol. 1994;24(4):1007-1009.

30. Steinbrink K, Wolfl M, Jonuleit H, Knop J, Enk AH Induction of tolerance by IL-10-treated dendritic cells. J Immunol. 1997;159(10):4772-4780.

31. Day CL, et al. PD-1 expression on HIV-specific T cells is associated with T-cell exhaustion and disease progression. Nature. 2006;443(7109):350-354.

32. Fuse S, Molloy MJ, Usherwood EJ. Immune responses against persistent viral infections: possi- ble avenues for immunotherapeutic interventions. Crit Rev Immunol. 2008;28(2):159-183.

33. Ogasawara K, Lanier LL. NKG2D in NK and T cell-mediated immunity. J Clin Immunol. 2005; 25(6):534-540.

34. Shan M, et al. HIV-1 gp120 mannoses induce immunosuppressive responses from dendritic cells. PLoS Pathog. 2007;3(11):e169.

35. Martinic MM, von Herrath MG. Novel strategies to eliminate persistent viral infections. Trends Immunol. 2008;29(3):116-124.

36. Moore KW, de Waal Malefyt R, Coffman RL, O'Garra A. Interleukin-10 and the interleukin-10 receptor. Annu Rev Immunol. 2001;19:683-765.

37. Mavilio D, et al. Characterization of CD56-/CD16+ natural killer (NK) cells: a highly dysfunctional NK subset expanded in HIV-infected viremic individuals. Proc Natl Acad Sci U S A. 2005;102(8):2886-2891.

38. Alter G, et al. Sequential deregulation of NK cell subset distribution and function starting in acute HIV-1 infection. Blood. 2005;106(10):3366-3369.

39. Meier A, et al. Upregulation of PD-L1 on monocytes and dendritic cells by HIV-1 derived TLR ligands. AIDS. 2008;22(5):655-658.

40. Lombardi V, Van Overtvelt L, Horiot S, Moingeon P. Human dendritic cells stimulated via TLR7 and/ or TLR8 induce the sequential production of Il-10, IFN-gamma, and IL-17A by naive CD4+ T cells. J Immunol. 2009;182(6):3372-3379.

41. Ejrnaes $M$, et al. Resolution of a chronic viral infection after interleukin-10 receptor blockade. J Exp Med. 2006;203(11):2461-2472.

42. Stacey AR, et al. Induction of a striking systemic cytokine cascade prior to peak viremia in acute human immunodeficiency virus type 1 infection, in contrast to more modest and delayed responses in acute hepatitis B and C virus infections. J Virol.2009; 83(8):3719-3733. 